\title{
Strong Duschinsky Mixing Induced Breakdown of Kasha's Rule in an Organic Phosphor
}

Lopa Paul, ${ }^{1}$ Torsha Moitra, ${ }^{2}$ Kenneth Ruud, ${ }^{3, *}$ and Swapan Chakrabarti. ${ }^{1, *}$

${ }^{1}$ Department of Chemistry, University of Calcutta

92, A.P.C.Road, Kolkata 700 009,India.

${ }^{2}$ Department of Chemistry, Technical University of Denmark,

Kemitorvet, 2800 Kgs. Lyngby, Denmark.

${ }^{3}$ Hylleraas Centre for Quantum Molecular Sciences, Department of Chemistry, University of

Troms $\varnothing$ - The Arctic University of Norway,

N-9037 Tromsø, Norway

AUTHOR INFORMATION

Corresponding Author

*E-mail: swcchem@ caluniv.ac.in Fax: 91-33-23519755

*E-mail: kenneth.ruud@uit.no 
ABSTRACT:We present the novel observation that Duschinsky mixings can lead to the breakdown of Kasha's rule in a white light phosphor molecule, Dibenzo[b,d]thiophen-2-yl (4chlorophenyl)methanone. Our theoretical analyses show the energy gap between the $\mathrm{T}_{1}$ and $\mathrm{T}_{2}$ states $(0.48 \mathrm{eV})$ is too large to allow for any significant population of the $\mathrm{T}_{2}$ state at room temperature and instead the faster intersystem crossing (ISC) between the $\mathrm{S}_{1}$ and $\mathrm{T}_{2}$ states is rather due to strong Duschinsky mixing induced, leading to the emission from the $\mathrm{T}_{2}$ state as well. A second-order cumulant-based method has been used for the calculation of the ISC rate, which suggests two order of magnitudes faster ISC rates for $S_{1} \rightarrow T_{2}$ compared to $S_{1} \rightarrow T_{1}$. We found that the carbonyl moiety of the $S_{1}$ and $T_{2}$ states of the molecule is significantly different with respect to bond angle and dihedral angles, engendering large displacements in selective normal modes, thus giving rise to strong Duschinsky mixing.

\section{TOC GRAPHICS}

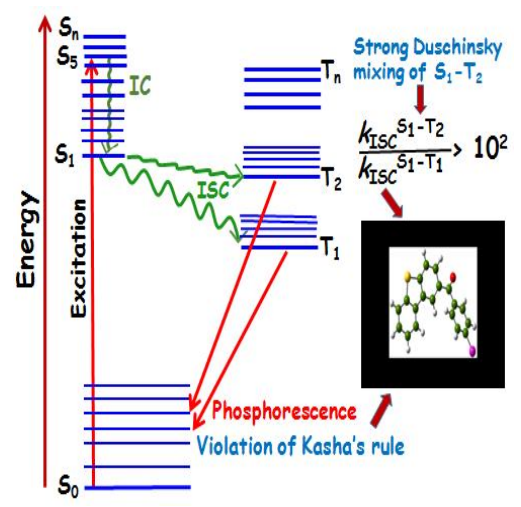


Single molecule white light emitters (SMWLEs) have recently been attracting attention in the field of white-light emitting devices owing to the easy device fabrication process, absence of color-aging and improved reproducibility compared to its contemporary multi-component emitters. ${ }^{1-6}$ To date, there are few examples of pure organic phosphorescent which emits solely from triplet excitons, ${ }^{4-6}$ because of weak spin-orbit coupling (SOC) and the quenching of triplet excitons. Moreover, to exhibit white-light phosphorescence, molecules need to cover the entire range of visible spectrum, which is possible if the phosphor displays dual emission involving a higher triplet state, usually the $T_{2}$ state, together with the lowest triplet state $\left(T_{1}\right) \cdot{ }^{7,8}$ Either strong intersystem crossing (ISC) or thermal population from the $\mathrm{T}_{1}$ state leads to $\mathrm{T}_{2}$ phosphorescence where the latter situation is favored by a smaller gap. ${ }^{8-11}$

In 2017, He et al. ${ }^{12}$ have reported some pure organic molecule showing dual room temperature phosphorescence (RTP), and interestingly one of these phosphors, Dibenzo[b,d]thiophen-2-yl (4chlorophenyl)methanone [ClBDBT], emit white light. They assumed that the small energy difference between the $T_{1}$ and $T_{2}$ states of CIBDBT allowed for thermal activation of the $T_{2}$ state from the $\mathrm{T}_{1}$, and finally emission from the both states results in the observed dual-emission peak. Here, we present an extensive theoretical analysis employing time-dependent density functional theory-based response theory (TDDFT-RT) ${ }^{13-16}$ to probe the dual-peak nature of CIBDBT or, more precisely, to scrutinize the reason for the population of the phosphorescence emitting $T_{2}$ state. We have calculated the rate of ISCs $\left(k_{\mathrm{ISCs}}\right)$ explicitly using the Condon approximation to the Fermi Golden rule within the framework of the time-dependent second-order cumulant expansion approach that includes Duschinsky mixing effect. ${ }^{17}$ Furthermore, the vibronic contribution of the two triplet states $\left(\mathrm{T}_{1}\right.$ and $\left.\mathrm{T}_{2}\right)$ and the corresponding phosphorescence lifetimes $(\tau)^{18}$ for the two states are also computed. 
The structure of the organic molecule CIBDBT is shown in Figure 1. Density functional theory (DFT) was used to optimize the ground-state $\left(\mathrm{S}_{0}\right)$ and the lowest excited triplet state $\left(\mathrm{T}_{1}\right)$ of CIBDBT, where the latter state was optimized from the $S_{0}$ optimized structure using an unrestricted approach. The lowest excited singlet state $\left(\mathrm{S}_{1}\right)$ and the upper excited triplet state $\left(\mathrm{T}_{2}\right)$ geometries were optimized from the electron density of the $S_{0}$ state and the $T_{1}$ state, respectively, with excited-state gradients obtained from TD-DFT method. All optimizations were performed at the Grimme's dispersion corrected B3LYP/6-311G(d,p) level of theory. To verify that the geometries correspond the energy minima, harmonic vibrational frequencies were calculated at the optimized structures to ensure there were no negative frequencies. CIBDBT has $\mathrm{C}_{1}$ symmetry and the bond parameters for the different states are collected in the Supporting Information, Table S1. The absorption calculations were carried out at the optimized geometry of the $\mathrm{S}_{0}$ state and the details are provided in the Supporting Information. All the geometry optimizations were carried out using the Gaussian 09 program. ${ }^{19}$

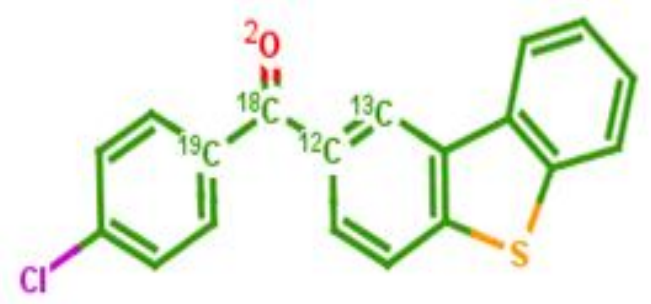

Figure 1. Structure of Dibenzo[b,d]thiophen-2-yl (4-chlorophenyl)methanone [CIBDBT]. The oxygen and four carbon atoms are labeled as the geometric parameters involving these atoms display noteworthy deviation in the excited states, specifically between $S_{1}$ and $T_{2}$ states.

The experimental report ${ }^{12}$ implies the absence of fluorescence from CIBDBT crystalline powders, which in turn suggests $S_{1}$ to be a dark state whereas the $T_{1} \rightarrow S_{0}$ and $T_{2} \rightarrow S_{0}$ phosphorescence wavelengths at $300 \mathrm{~K}$ have been observed at $\sim 570 \mathrm{~nm}$ and $\sim 470 \mathrm{~nm}$, 
respectively.The calculated TDDFT fluorescence strength for the $\mathrm{S}_{1} \rightarrow \mathrm{S}_{0}$ transition agrees with the experimental observation. To obtain the phosphorescence wavelengths, we did TDDFT-RT computations at the $\mathrm{T}_{1}$ geometry at the B3LYP-D3/Sadlej-pVTZ level of theory as implemented in the Dalton program. ${ }^{20}$ The $\mathrm{T}_{1}$ emission occurs at $566 \mathrm{~nm}(2.19 \mathrm{eV})$ and that of $\mathrm{T}_{2}$ at $464 \mathrm{~nm}$ $(2.67 \mathrm{eV})$, in good agreement with experiment. A comparison of the calculated spectrum with the experimental spectrum excluding vibrational coupling is shown in Figure 2.

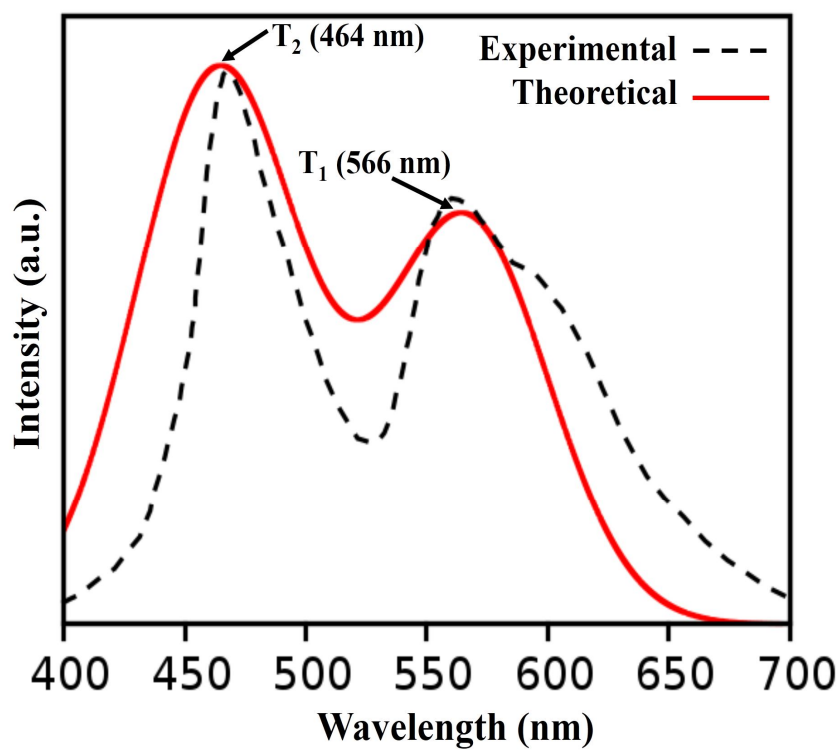

Figure 2. Theoretically simulated phosphorescence spectrum with FWHM $=1000 \mathrm{~cm}^{-1}$ (red) and the experimental phosphorescence (dashed) of ClBDBT.

It is well known that broad spectra covering the entire visible region have to have vibronic contributions. ${ }^{21-23} \mathrm{He}$ et al ${ }^{12}$ did take this into consideration and calculated the vibrationally resolved spectra for the $T_{1}$ and $T_{2}$ emissions. For the vibronic spectrum of the $T_{1}$ state, they correlated the lowest wavelength peak of the four peaks originating from this transition with the experimentally observed sharp peak at lower temperature (>250 K) and assigned it as the $0-0$ peak, which remained smeared due to thermal broadening at high temperature. For $\mathrm{T}_{2}$, they 
obtained two vibrationally resolved peaks of comparable intensity and designated these as $0-1$ peaks. The TDDFT-RT calculations are based on purely electronic states, so in order to investigate the role of vibronic coupling in both the triplet states, we have calculated the FranckCondon (FC) overlap integral involving all the 93 normal modes of ClBDBT and considered five vibrational quanta for each normal mode (details are given in the Supporting Information). Figure 3a represents the FC vibronic spectrum of the $T_{1}$ and $T_{2}$ states. From Figure $3 a$, it is clear that the $T_{1}$ state has four vibrationally resolved peaks covering the green and red region of the visible spectrum and the $T_{2}$ state displays two intense peaks in the blue part of the spectrum, in addition to three less intense peaks. Figure $3 \mathrm{a}$ also shows that the theoretically simulated spectra match that of He et al. ${ }^{12}$ and in line with their study, we label the 0-0 peak of the $\mathrm{T}_{1}$ state as the one obtained at $512 \mathrm{~nm}$, blue-shifted by $54 \mathrm{~nm}$ from the major peak at $566 \mathrm{~nm}$. He et al. ${ }^{12}$ assigned the rest of the peaks as 0-1, 0-2 and 0-3 occuring at a single vibrational mode of 1731 $\mathrm{cm}^{-1}$. However, we are not able to reproduce these results, as the longer wavelength peaks are of very high frequency and are likely to occur from the mixing of two or more normal modes of vibration. As far as $\mathrm{T}_{2}$ is concerned, our vibrationally resolved spectra differ from that of $\mathrm{He} e t$ $a l .{ }^{12}$ These differences could be due to the choice of the level of theory used. Moreover, we also conducted a temperature probe on the FC spectrum of the two above mentioned triplet states (Figure $3 \mathrm{~b}$ and $3 \mathrm{c}$ ) using FCclasses. ${ }^{24}$ On lowering the temperature, an increase in the intensities of the vibronic spectra of both states $\left(\mathrm{T}_{1}\right.$ and $\left.\mathrm{T}_{2}\right)$ is observed. The increase in the intensities is attributed to the gain in the quantum yield due to lowering of temperature. 

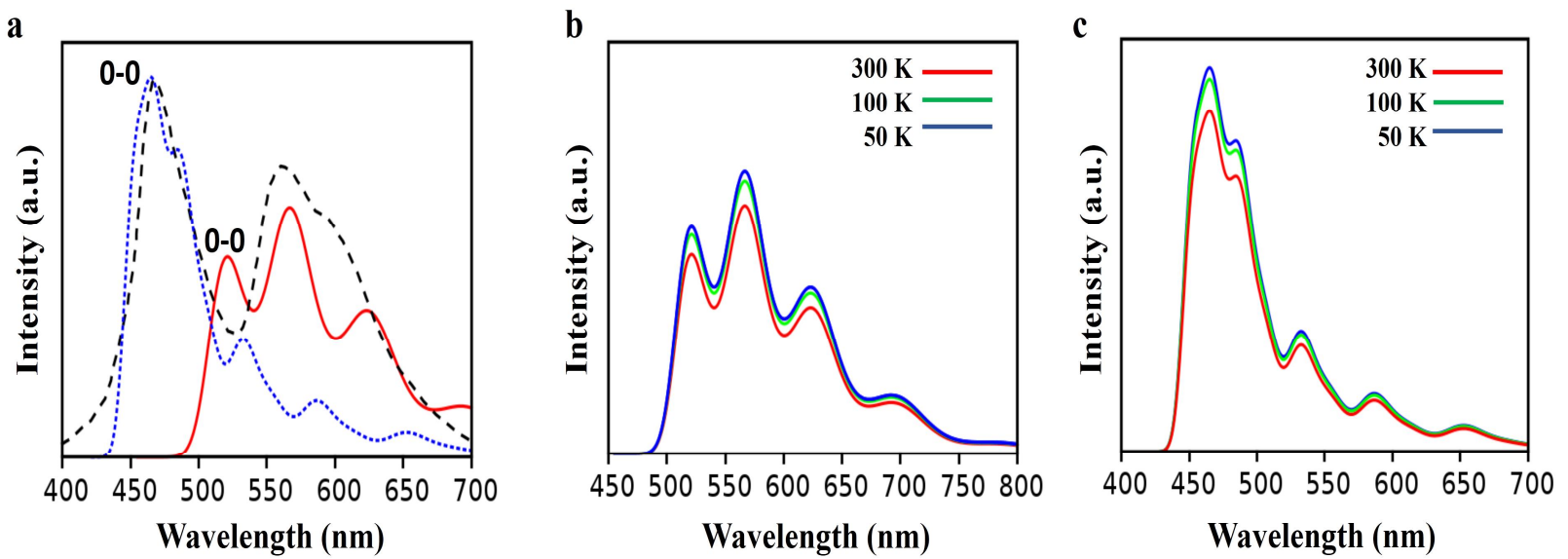

Figure 3. (a)Vibrationally resolved simulated spectrum with $\mathrm{FWHM}=1000 \mathrm{~cm}^{-1}$ (red line forT state and blue dotted line for $T_{2}$ state) indicating the $0-0$ transition together with the experimental photophosphorescence spectrum (black dashed line). (b)Vibrationally resolved simulated spectrum with $\mathrm{FWHM}=1000 \mathrm{~cm}^{-1}$ for the $\mathrm{T}_{1}$ state at different temperature. (c)Vibrationally resolved simulated spectrum with $\mathrm{FWHM}=1000 \mathrm{~cm}^{-1}$ for the $\mathrm{T}_{2}$ state at different temperature. The experimental phosphorescence showed peaks at $\sim 470 \mathrm{~nm}\left(\mathrm{~T}_{2} \rightarrow \mathrm{S}_{0}\right)$ and at $\sim 570 \mathrm{~nm}\left(\mathrm{~T}_{1} \rightarrow \mathrm{S}_{0}\right)$.

As per earlier reports, $T_{2}$ phosphorescence appears to be more promising in molecules exhibiting low $\mathrm{T}_{1}-\mathrm{T}_{2}$ energy gap where the higher triplet states are mostly populated from thermal activation of the lower triplet state (i.e. $\left.\mathrm{T}_{1}\right) \cdot{ }^{8-10}$ Unfortunately the large energy gap obtained for $\operatorname{ClBDBT}\left(\Delta \mathrm{E}_{\mathrm{T}_{2}-\mathrm{T}_{1}}=0.48 \mathrm{eV}\right)$, prevents sufficient Boltzmann population of the $\mathrm{T}_{2}$ state even at room temperature, thus indicating faster $\mathrm{S}_{1} \rightarrow \mathrm{T}_{2}$ intersystem crossing (ISC) as the probable source of population gain in the $T_{2}$ state. The rate of ISC serves as the fundamental step towards phosphorescence and can be evaluated within the frameworkof time-dependent perturbation theory using Fermi's Golden rule. ${ }^{17}$ Applying the FC approximation and using the Heisenberg picture for the SOC Hamiltonian, Marian et al. ${ }^{25,26}$ have proposed three different formulae for 
calculating the rate of ISC $\left(k_{\mathrm{ISC}}\right)$. Recently, Moitra et al. ${ }^{17}$ have utilized Marian's ${ }^{25,26}$ cumulant expansion-based method for calculating the $k_{\text {ISC }}$ and the details of the method is provided in the Supporting Information. The simplified expression for $k_{\mathrm{ISC}}$ is given as

$$
k_{\text {Isc }}=4 \pi\left\langle\mathrm{T}_{\mathrm{n}}\left|\mathrm{H}_{\mathrm{soc}}\right| \mathrm{S}_{1}\right\rangle^{2} \times \exp \left(-\kappa_{2}^{\mathrm{R}, \mathrm{TI}}\right) \int_{0}^{+\infty} \cos \left(\kappa_{1} \mathrm{t}+\kappa_{2}^{\mathrm{Im}}\right) \exp \left(-\kappa_{2}^{\mathrm{R}, \mathrm{TD}}\right) \mathrm{dt}
$$

Equation (1) is obtained by separating the real and imaginary part of the cumulants. ${ }^{17,25,26}$ The first term provides the square of the SOC matrix elements for the $S_{1} \rightarrow T_{n}$ transition $\left(<\mathrm{T}_{1}\left|\mathrm{H}_{\mathrm{SOC}}\right| \mathrm{S}_{1}\right\rangle=25 \mathrm{~cm}^{-1}$ and $\left.\left\langle\mathrm{T}_{2}\left|\mathrm{H}_{\mathrm{SOC}}\right| \mathrm{S}_{1}\right\rangle=29 \mathrm{~cm}^{-1}\right)$, whereas the second term consists of the real time-independent $\left(\kappa^{\mathrm{R}, \mathrm{TI}}\right)$ part, the real time-dependent $\left(\kappa^{\mathrm{R}, \mathrm{TD}}\right)$ part and the imaginary $\left(\kappa^{\mathrm{Im}}\right)$ part of the cumulant expansion, which inturn contains the Duschinsky rotation matrix $(\mathrm{J})$ and the displacement vector $(\mathrm{D})$ connecting the normal modes of triplet $\left(\mathrm{Q}_{\mathrm{T}}\right)$ and $\operatorname{singlet}\left(\mathrm{Q}_{\mathrm{S}}\right)$ states. The SOC matrix elements are calculated using the zero-order regular approximation (ZORA) as implemented in ADF $2016 .{ }^{27}$ To ensure the convergence of the infinitely oscillating function, we have added a Gaussian damping function within the integral of equation 1 .The $k_{\text {ISC }}$ thus achieved from equation 1 , for $\mathrm{S}_{1}-\mathrm{T}_{1}$ and $\mathrm{S}_{1}-\mathrm{T}_{2}$ are $7.71 \times 10^{8} \mathrm{~s}^{-1}$ and $2.13 \times 10^{11} \mathrm{~s}^{-1}$, respectively, with the latter being $\sim 10^{2}$ faster than the former (Table 1). Although the SOC values for the $S_{1}-T_{1}$ and $S_{1}$ $\mathrm{T}_{2}$ transitions are comparable, the smaller energy separation $(0.09 \mathrm{eV})$ between the $\mathrm{S}_{1}$ and $\mathrm{T}_{2}$ state favors more efficient ISC between these states. The other factor that could be important in determining the net ISC rate is the Duschinsky mixing between the normal modes of the two electronic states. The presence of significant off-diagonal elements in the plot for the Duchinsky rotation matrix corresponding to the $S_{1} \rightarrow T_{n}(n=1,2)$ non-radiative transitions (Figure 4a) exhibits strong mixing of the vibrational states involving all the 93 normal modes. In particular, Figure $4 \mathrm{a}$ shows that the extent of state mixing is quite strong for $S_{1} \rightarrow T_{1}$ and even stronger for $S_{1}-T_{2}$. To 
get insight into the origin of this strong Duschinsky mixing, we have carefully inspected the geometryof all the electronic states, $\mathrm{S}_{1}, \mathrm{~T}_{1}$ and $\mathrm{T}_{2}$. From Table $\mathrm{S} 1$ of the Supporting Information, it is clear that the bond lengths connecting the heavy atoms do not change upon excitation. However, the bond angles and dihedral angles involving the oxygen atom reveal noteworthy differences, in particular between the $\mathrm{S}_{1}$ and $\mathrm{T}_{2}$ geometries. The bond angles $<\mathrm{O}_{2}-\mathrm{C}_{18}-\mathrm{C}_{12}$ and $<\mathrm{O}_{2}-\mathrm{C}_{18}-\mathrm{C}_{19}$ show an increment of $\sim 7^{\circ}$ and a decrease by $\sim 9^{\circ}$ in the $\mathrm{T}_{2}$ geometry, respectively, with respect to the $\mathrm{S}_{1}$ state, while the angles connecting only the carbon atoms, i.e., $<\mathrm{C}_{12}-\mathrm{C}_{18}-\mathrm{C}_{19}$ and $<\mathrm{C}_{13}-\mathrm{C}_{12}-\mathrm{C}_{18}$ display negligible change between these states. Moreover, a considerably large change ranging from $\sim 23^{\circ}-\sim 31^{\circ}$ is observed in the five dihedral angles - four involving oxygen atom, and one linking only the carbon atoms $\left(<\mathrm{C}_{13}-\mathrm{C}_{12}-\mathrm{C}_{18}-\mathrm{C}_{19}\right)$ between the geometry of the $\mathrm{S}_{1}$ and $T_{2}$ states. This significant change in the geometry of the $S_{1}$ and $T_{2}$ states suggests that large normal mode displacement vectors will lead to strong Duschinsky mixing between the two states. It is worth noting that we have used Cartesian coordinates instead of the curvilinear one and this is justified since the frequencies of the normal modes involving all the above stated bond angles and dihedral angles of the two states are greater than $100 \mathrm{~cm}^{-1}$ (falling in the range 300$\left.900 \mathrm{~cm}^{-1}\right)$.

To check the robustness of our calculations, a variety of $k_{\mathrm{ISCs}}$ have been calculated for the abovementioned transitions, changing different parameters affecting the calculation (number of points in the integration, damping factor and final time of integration) and the results obtained were found to be very consistent (Table S3). Moreover, the variation in the real part of the cumulant expansion as a function of time for $\mathrm{S}_{1}-\mathrm{T}_{1}$ and $\mathrm{S}_{1}-\mathrm{T}_{2}$ transitions are presented in Figure $4 \mathrm{~b}$. Figure $4 \mathrm{~b}$ reflects the proper convergence of the integral for the $\mathrm{S}_{1} \rightarrow \mathrm{T}_{1}$ transition. The decay time is atleast an order of magnitude smaller than the final time of integration. Additionally, the decay 
time is set such that it is not less than the time corresponding to the lowest vibrational frequency of the states involved. This ensures that contributions from all vibrational modes towards the ISC is taken into account. It is also important to notice that the oscillating function for the $S_{1} \rightarrow T_{2}$ transition is damped completely even in the absence of a gaussian damping function. This can be attributed to the large normal mode displacement between the states involved in the transition. ${ }^{25}$
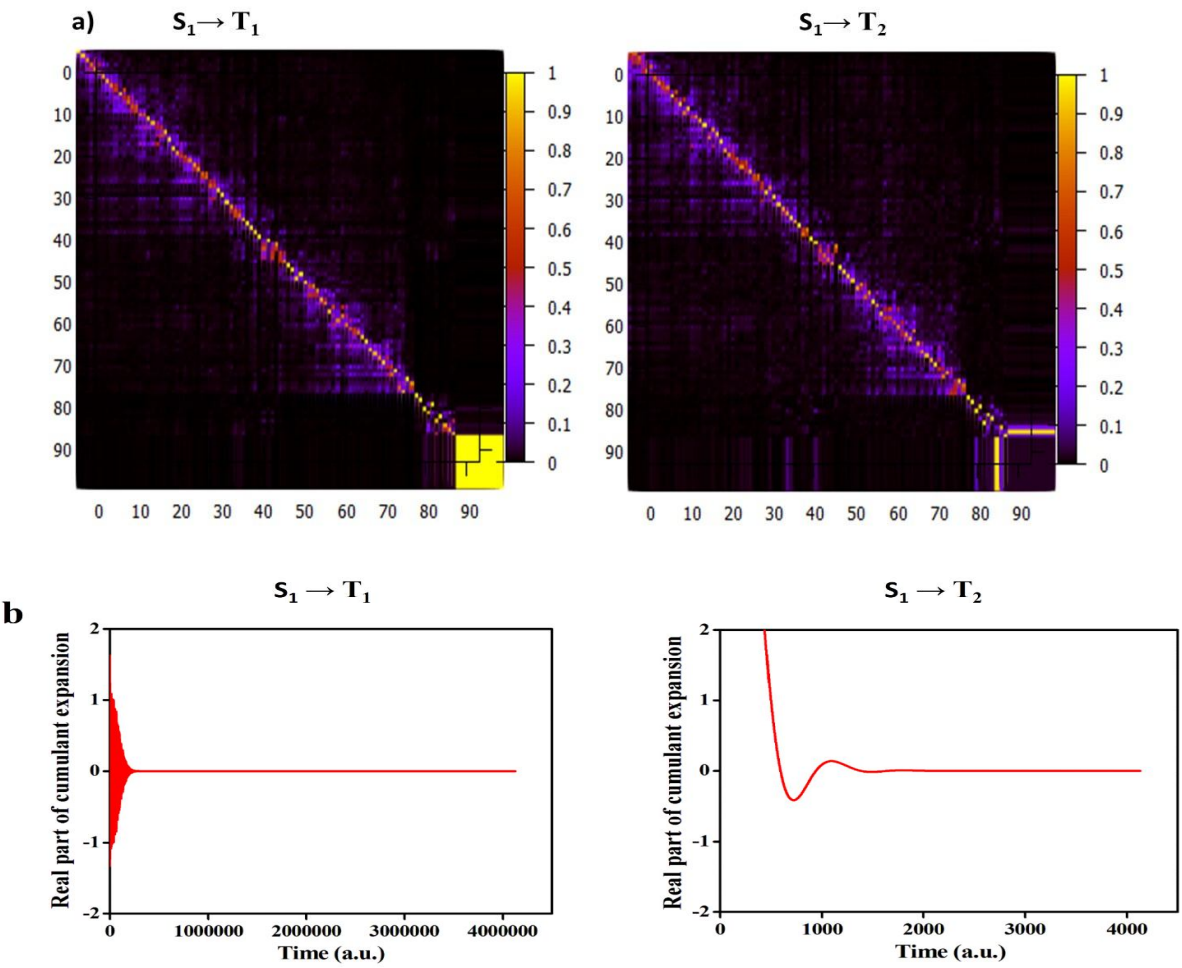

Figure 4. (a) Absoulute vaules of Duschinsky rotation matrix for $S_{1} \rightarrow T_{1}$ (left) and $S_{1} \rightarrow T_{2}$ (right) transition. (b) The real part of the cumulant expansion of $S_{1} \rightarrow T_{1}($ left $)$ transition for the complete time range of integration (damping factor=2.0, final time of integration=100 ps, no. of points of integration=100000) and $\mathrm{S}_{1} \rightarrow \mathrm{T}_{2}$ (right) transition as a function of time for the first $100 \mathrm{fs}$ with no damping. 
Table1. Electronic transition, transition energy $[\mathrm{E}(\mathrm{eV})], \mathrm{FC}$ weighted radiative rate constant $\left[k_{\mathrm{r}}^{0-}\right.$ $\left.{ }^{0}\left(\mathrm{~s}^{-1}\right)\right]$, non-radiative rate constant $\left[k_{\mathrm{nr}}\left(\mathrm{s}^{-1}\right)\right]$,total computed lifetime $\left[\tau_{\mathrm{n}}^{\text {comp }}(\mathrm{ms})\right]$ and the experimental lifetime $\left[\tau_{\mathrm{n}}{ }^{\text {expm }}(\mathrm{ms})\right]$ of ClBDBT.

\begin{tabular}{|c|c|c|c|c|c|}
\hline $\mathrm{T}_{\mathrm{n}} \rightarrow \mathrm{S}_{0}$ & $\mathrm{E}$ & $k_{\mathrm{r}}^{0-0}$ & $k_{\mathrm{nr}}$ & $\tau_{\mathrm{n}}^{\text {comp }}$ & $\tau_{\mathrm{n}}^{\text {expm }}$ \\
\hline $\mathrm{n}=1$ & 2.19 & $4.57 \times 10^{-2}$ & 4.28 & 231 & 123.4 \\
\hline $\mathrm{n}=2$ & 2.67 & $9.49 \times 10^{-4}$ & 111.13 & 8.90 & 0.41 \\
\hline
\end{tabular}

The faster $k_{\mathrm{ISC}}$ for $\mathrm{S}_{1} \rightarrow \mathrm{T}_{2}$ with respect to that of $\mathrm{S}_{1} \rightarrow \mathrm{T}_{1}$ is further validated from the natural transition orbitals (NTOs) depicted in Figure 5. The benzophenone motif exhibits (n- $\left.\pi^{*}\right)$ character and the dibenzothiophene moiety $\left(\pi-\pi^{*}\right)$ character. Interestingly, the $S_{1} \rightarrow T_{1}$ transition is of ${ }^{1}\left[n-\pi^{*}\right] \rightarrow{ }^{3}[n-\pi]^{*}$ type, whereas the $S_{1} \rightarrow T_{2}$ transition involves ${ }^{1}\left[n-\pi^{*}\right] \rightarrow^{3}\left[\pi-\pi^{*}\right]$ orbitals.

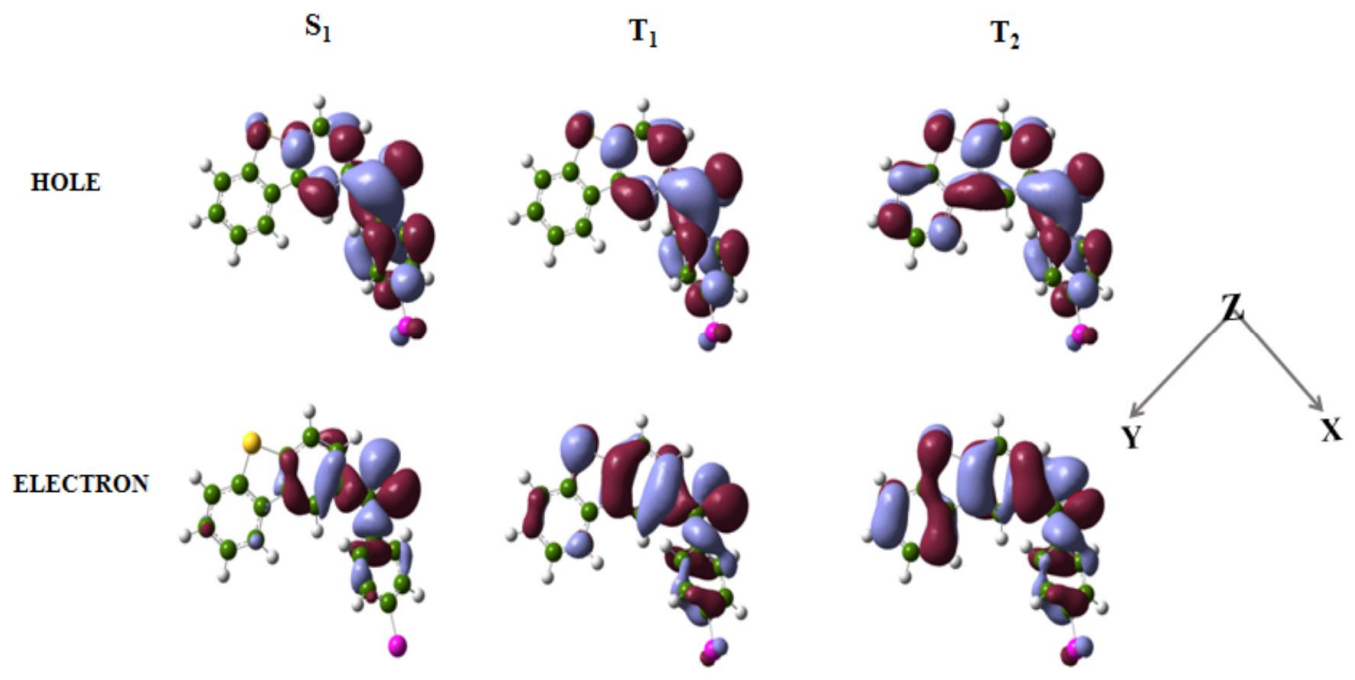

Figure 5. Natural transition orbitals (NTO) of $S_{1}$ (left), $T_{1}$ (centre) and $T_{2}$ (right) states of CIBDBT. Isovalue $=0.02$. 
The above observation thus helps rationalize the observed phosphorescence from the $\mathrm{T}_{2}$ state, and together with the phosphorescence from the $\mathrm{T}_{1}$ state this gives rise to the dual peak spectrum. The respective lifetimes of the two states as observed by He et al. ${ }^{12}$ are $123.4 \mathrm{~ms}$ for $\mathrm{T}_{1}$ and 0.41 $m s$ for $T_{2}$. We have explicitly evaluated both the radiative and nonradiative rate constants for the two states. The total lifetime $(\tau)$ is expressed as $\tau=\left(k_{\mathrm{r}}+k_{\mathrm{nr}}\right)^{-1}$, where $k_{\mathrm{r}}$ and $k_{\mathrm{nr}}$ are the radiative and nonradiative rate constants, respectively. $k_{\mathrm{r}}$ is computed utilizing the high-temperature limit approximation ${ }^{28}$ where all the three substates of the triplet manifold contribute equally in the phosphorescence process, and the relevant equation is specified in the Supporting Information. Additionally, to account for vibronic coupling, the 0-0 FC overlap integrals (0-0 FC factor for $\mathrm{T}_{1} \rightarrow \mathrm{S}_{0}=1.7625 \times 10^{-3}$ and for $\mathrm{T}_{2} \rightarrow \mathrm{S}_{0}=1.0629 \times 10^{-6}$ ) are considered in the evaluation of $k_{\mathrm{r}}$. The $k_{\mathrm{r}}$ achieved for $\mathrm{T}_{1}-\mathrm{S}_{0}$ and $\mathrm{T}_{2}-\mathrm{S}_{0}$ are $4.57 \times 10^{-2} \mathrm{~s}^{-1}$ and $9.49 \times 10^{-4} \mathrm{~s}^{-1}$, respectively.The rate constants with the 0-0 FC correction $\left(k_{\mathrm{r}}^{0-0}\right.$ and $\left.k_{\mathrm{nr}}\right)$, together with the computed $\left(\tau_{\mathrm{n}}^{\text {comp }}\right)$ and experimental lifetimes $\left(\tau_{\mathrm{n}}^{\text {exmp }}\right)$ are presented in Table 1 .

$k_{\mathrm{nr}}$ is calculated using Marcus-Levich-Jortner theory ${ }^{29}$ derived in the framework of Fermi's golden rule, and is expressed as s $^{30,31}$

$k_{\mathrm{nr}}=\frac{2 \pi}{\hbar}\left\langle\mathrm{T}_{\mathrm{n}}\left|H_{s o c}\right| \mathrm{S}_{0}\right\rangle^{2} \times \mathrm{FCWD}$

where the first term represents the square of the spin-orbit coupling (SOC) matrix element and the second term denotes the Franck-Condon-weighted density of states (FCWD). The details of equation (2) are given in the Supporting Information. The SOCs are calculated with the same method as mentioned earlier, and the values are $\left\langle\mathrm{T}_{1}\left|\mathrm{H}_{\mathrm{SOC}}\right| \mathrm{S}_{0}\right\rangle=54 \mathrm{~cm}^{-1}$ and $\left\langle\mathrm{T}_{2}\left|\mathrm{H}_{\mathrm{SOC}}\right| \mathrm{S}_{0}\right\rangle=34$ $\mathrm{cm}^{-1}$ for the $\mathrm{T}_{1} \rightarrow \mathrm{S}_{0}$ and $\mathrm{T}_{2} \rightarrow \mathrm{S}_{0}$ emissions, respectively, the former being only 1.5 times greater than the latter. The FCWD depends on various energy parameters, reorganization energy, normal 
mode frequency and the energy difference between the states of interest as well as on the dimensionless electron-phonon coupling strength (the Huang-Rhys factor $\left.(S)^{32}\right)$ over all the normal modes (Eq S10 in the Supporting Information).We used FCclasses ${ }^{24}$ to calculate all these parameters and finally obtained $k_{\mathrm{nr}}$ for $\mathrm{T}_{1}\left(4.28 \mathrm{~s}^{-1}\right)$ and $\mathrm{T}_{2}\left(111.13 \mathrm{~s}^{-1}\right)$, where the value of $\mathrm{T}_{2}$ is almost $\sim 26$ times greater than $\mathrm{T}_{1}$. This observation is indicative of the decisive role played by FCWD in the estimation of $k_{\mathrm{nr}}$. Overall, such low rate of non-radiative transition is due to the large reorganization energy $(\lambda)$ involved in the emission procedure; for $T_{1} \rightarrow S_{0}$, we considered $\lambda$ of both states $\left(\lambda_{\mathrm{T}_{1}+\mathrm{S}_{0}}=1.72 \mathrm{eV}\right)$, whereas for the $\mathrm{T}_{2} \rightarrow \mathrm{S}_{0}$ transition, we used $\lambda$ of the ground state only $\left(\lambda_{\mathrm{s}_{0}}=1.10 \mathrm{eV}\right)$. The low non-radiative rates contributes to the fact that the lifetimes are in the millisecond range. From Table 1 , we find $\tau_{1}{ }^{\text {comp }}=231 \mathrm{~ms}$ and $\tau_{2}{ }^{\text {comp }}=8.90 \mathrm{~ms}$, the computed lifetimes corroborating nicely with the experimental data.

To conclude, we have established that the reason for phosphorescence from the $\mathrm{T}_{2}$ state of the single molecule CIBDBT, is the faster rate of $S_{1} \rightarrow T_{2}$ ISC, rather than the emission from thermally excited $\mathrm{T}_{2}$ state. The population gain of $\mathrm{T}_{2}$ from $\mathrm{S}_{1}$ helps achieve intense phosphorescence in the blue region, and the phosphorescence in the higher wavelength region occurs due to $\mathrm{T}_{1}$, leading to the emission of white-light and a dual-peak spectrum. Our study reveals that strong Duschinsky mixing between the normal modes of $\mathrm{S}_{1}$ and $\mathrm{T}_{2}$ is mainly attributed to the ultrafast ISC between these states. The meticulous analyses of the results obtained suggest that the bond angles and dihedral angles in the neighborhood of the carbonyl moiety of the molecule studied is significantly different, indicating that the displacement vectors in certain normal modes will be very large, which in turn is responsible for the very strong Duschinsky mixing in the normal modes of $\mathrm{S}_{1}$ and $\mathrm{T}_{2}$.

\section{ASSOCIATED CONTENT}




\section{Supporting Information.}

(1) Computational details. (2) Co-ordinates of the optimized geometries of CIBDBT. (3)

Structural parameters. (4) One photon absorption (OPA). (5) Evaluation of Franck-Condon (FC) integrals. (6) Analysis for intersystem crossing (ISC) rate constant [ $\left.k_{\mathrm{ISC}}\right]$. (7)Estimation of the radiative rate constant $\left(k_{\mathrm{r}}\right)$ for pure electronic transitions. (8) Analysis of the expression for the non-radiative rate constant $\left(k_{\mathrm{nr}}\right)$.(PDF)

\section{AUTHOR INFORMATION}

\section{Notes}

The authors declare no competing financial interests.

\section{ACKNOWLEDGMENT}

LP thanks the Council of Scientific and Industrial Research (CSIR) for granting her the Senior Research Fellowship. TM acknowledges support from the Marie Skłodowska-Curie European Training Network "COSINE - COmputational Spectroscopy In Natural sciences and Engineering”, Grant Agreement No. 765739. KR has received support from the Research Council of Norway through a Centre of Excellence Grant (Grant No 262695). Computer time was provided by theNorwegian Supercomputer Program NOTUR (Grant No. NN4654K).

\section{REFERENCES}

(1) Chen, Y. -H. et al. Insight into the Mechanism and Outcoupling Enhancement of Excimer-Associated White Light Generation. Chem. Sci. 2016, 7, 3556-3563.

(2) Zhang, Z.; Wu, Y. -S.; Tang, K. -C.; Chen, C. -L.; Ho, J. -W.; Su, J.; T, H.; C, P. -T. Excited-State Conformational/Electronic Responses of Saddle-Shaped N,N'-Disubstituted- 
Dihydrodibenzo[a,c]phenazines: Wide-Tuning Emission from Red to Deep Blue and White Light Combination. J. Am. Chem. Soc. 2015, 137, 8509-8520.

(3) Mao, Z.; Yang, Z.; Mu, Y.; Zhang, Y.; Wang, Y. F.; Chi, Z.; Lo, C. C.; Liu, S.; Lien, A.; $\mathrm{Xu}$, J. Linearly Tunable Emission Colors Obtained from a Fluorescent-Phosphorescent Dual-Emission Compound by Mechanical Stimuli. Angew. Chem. Int. Ed. 2015, 54, 62706273.

(4) Wong, K. M. -C.; Yam, V. W. -W. Self-Assembly of Luminescent Alkynylplatinum(II) Terpyridyl Complexes: Modulation of Photophysical Properties through Aggregation Behaviour. Acc. Chem. Res. 2017, 44, 424-434.

(5) Shao, S.; Ding, J.; Wang, L. Jing, X.; Wang, F. White Electroluminescence from AllPhosphorescent Single Polymers on a Fluorinated Poly(Arylene Ether Phosphine Oxide) Backbone Simultaneously Grafted with Blue and Yellow Phosphors. J. Am. Chem. Soc. 2012, 134, 20290-20293.

(6) Ni, W. -X.; Li, M.; Zheng, J.; Zhan, S, -Z.; Qiu, Y. -M.; Ng, S. W.; Li, D. Approaching White-Light Emission from a Phosphorescent Trinuclear Gold(I) Cluster by Modulating its Aggregation Behavior. Angew. Chem. Int. Ed. 2013, 52, 13472-13476.

(7) Chu, S. -Y.; Goodman, L. A Simple Theoretical Model for Dual Phosphorescence. Chem. Phys. Lett. 1975, 32, 24-27.

(8) Chaudhuri, D. et. al. Metal-Free OLED Triplet Emitters by Side-Stepping Kasha's Rule. Angew. Chem. Int. Ed. 2013, 52, 13449-13452.

(9) Wagner, P. J.; Kemppainen. A. E.; Schott, H. N. Effects of Ring Substituents on the Type II Photoreactions of Phenyl Ketones. How Interactions Between Nearby Excited Triplets Affect Chemical Reactivity. J. Am. Chem. Soc. 1973, 95, 5604-5614.

(10) Itoh, T. Successive Occurrence of the $T_{1}\left(\pi, \pi^{*}\right)$ and $T_{2}\left(n, \pi^{*}\right)$ Phosphorescence and the $\mathrm{S}_{1}\left(\mathrm{n}, \pi^{*}\right)$ Fluorescence Observed for p-Cyanobenzaldehyde in a Solid Matrix. J. Lumin. 2004, 109, 221-225. 
(11) Itoh, T. Fluorescence and Phosphorescence from Higher Excited States of Organic Molecules. Chem. Rev. 2012, 112, 4541-4568.

(12) He, Z.; Zhao, W.; Lam, J. W. Y.; Peng, Q.; Ma, H.; Liang, G.; Shuai, Z.; Tang, B. Z. White Light Emission from a Single Organic Molecule with Dual Phosphorescence at Room Temperature. Nat. Commun. 2017, 8:416, 1-8.

(13) Hettema, H.; Jensen, H. J.; Jørgensen, P.; Olsen, J. Quadratic Response Functions for a Multiconfigurational Self-Consistent Field Wave Functions. J. Chem. Phys. 1992, 97, 1174-1190.

(14) Vahtras, O.; Ågren, H.; Jørgensen, P.; Jensen, H. J. A.; Helgaker, T.; Olsen, J. Multiconfigurational Quadratic Response Functions for Singlet and Triplet Perturbations: The Phosphorescence Lifetime of Formaldehyde. J. Chem. Phys. 1992, 97, 9178-9187.

(15) Runge, E.; Gross, E. K. U. Density-Functional Theory for Time-Dependent Systems. Phys. Rev. Lett. 1984, 52, 997-1000.

(16) Salek, P.; Vahtras, O.; Helgaker, T.; Ågren, H. Density-functional Theory of Linear and Nonlinear Time-Dependent Molecular Properties. J. Chem. Phys. 2002, 117, 9630-9645.

(17) Moitra, T.; Alam. M. M.; Chakrabarti, S. Intersystem Crossing Rate Dependent Dual Emission and Phosphorescence from Cyclometalated Platinum Complexes: A Second Order Cumulant Expansion Based Approach. Phys. Chem. Chem. Phys. 2018, 20, 2324423251.

(18) Kühn, M.; Weigend, F. Phosphorescence Lifetimes of Organic Light-Emitting Diodes from Two-Component Time-Dependent Density Functional Theory. J. Chem. Phys. 2014, $141,224302$.

(19) GAUSSIAN 09, revision D.01, Gaussian, Inc.: Wallingford, CT, 2009.

(20) DALTON, a Molecular Electronic Structure Program, Release Dalton 2016.2 (2015), see http://daltonprogram.org. (22 Jan, 2018). 
(21) Kleinschmidt, M.; Wüllen, C. V.; Marian, C. M. Intersystem-Crossing and Phosphorescence Rates in fac-Ir ${ }^{\mathrm{III}}(\mathrm{ppy})_{3}$ : A Theoretical Study Involving Multi-Reference Configuration Interaction Wavefunctions. J. Chem. Phys. 2015, 142, 94301-94316.

(22) Marian, C. M. Spin-Orbit Coupling and Intersystem Crossing in Molecules. WIREs Comput Mol Sci 2012, 2, 187-203.

(23) Paul, L.; Chakrabarti, S.; Ruud, K. Anomalous Phosphorescence from an Organometallic White-Light Phosphor. J. Phys. Chem. Lett. 2017, 8, 4893-4897.

(24) Santoro, F. FCclasses, a Fortran 77 code : http://village.ipcf.cnr.it (22 June, 2018).

(25) Etinski, M.; Tatchen, J.; Marian, C. M. Time-Dependent Approaches for the Calculaiton of Intersystem Crossing Rates. J. Chem. Phys. 2011, 134, 154105-154114.

(26) Föller, J.; Kleinschmidt, M.; Marian, C. M. Phosphorescence or Thermally Activated Delayed Fluorescence? Intersystem Crossing and Radiative Rate Constants of a ThreeCoordinate Copper(I) Complex Determined by Quantum-Chemical Methods. Inorg. Chem. 2016, 55, 7508-7516.

(27) ADF2016; SCM, Theoretical Chemistry, Virje Universiteit: Amsterdam, The Netherlands. http://www.scm.com. (21 June, 2018).

(28) Younker, J. M.; Dobbs, K. D. Correlating Experimental Photophysical Properties of Iridium(III) Complexes to Spin-Orbit Coupled TDDFT Predictions. J. Phys. Chem. C 2013, 117, 25714-25723.

(29) Brédas, J. L.; Beljonne, D.; Coropceanu, V.; Cornil, J. Charge-Transfer and EnergyTransfer Processes in Pi-Conjugated Oligomers and Polymers: A Molecular Picture. Chem. Rev. 2004, 104, 4971-5003.

(30) Tong, G. S. M.; Chan, K. T.; Chang, X.; Che, C. Theoretical Studies on the Photophysical Properties of Luminescent PinserGold(III) Arylacetylide Complexes: The Role of $\pi$-Conjugation at the C-Deprotonated $\left[\mathrm{C}^{\wedge} \mathrm{N}^{\wedge} \mathrm{C}\right]$ Ligand. Chem. Sci. 2015, 6, 30263037. 
(31) Schimdt, K.et al. Intersystem Crossing Processes in Nonplanar Aromatic Heterocyclic Molecules. J. Phys. Chem. A 2007, 111,10490-10499.

(32) Jong, M. D.; Seijo, L.; Meijerink, A.; Rabouw, F. T. Resolving the Ambiguity in the Relation between Stokes Shift and Huang-Rhys Parameter. Phys. Chem. Chem. Phys. 2015, 17, 6959-16969. 